\title{
Neutron Strain Scanning of Duplex Steel Subjected to 4-Point-Bending with Particular Regard to the Strain Free Lattice Parameter $D_{0}$
}

\author{
S. Pulvermacher ${ }^{1, a}$, J. Gibmeier ${ }^{1, b}$, J. Saroun ${ }^{2, c}$, J. Rebelo Kornmeier ${ }^{3, d}$, \\ F. Vollert ${ }^{1, e}$ and T. Pirling ${ }^{4, f}$
}

${ }^{1}$ Karlsruher Institut für Technologie, IAM-WK, Kaiserstraße 12, 76131 Karlsruhe, Germany

${ }^{2}$ Nuclear Physics Institute of the ASCR, 25068 Řež, Czech Republic

${ }^{3}$ Heinz Maier-Leibnitz Zentrum (MLZ), TU München, D-85748 Garching, Germany

${ }^{4}$ Institut Laue-Langevin, 38042 Grenoble, France

asamuel.pulvermacher@kit.edu, bjens.gibmeier@kit.edu, 'saroun@ujf.cas.cz,

djoana.kornmeier@frm2.tum.de, ${ }^{\mathrm{e}}$ florian.vollert@kit.edu, ${ }^{f}$ pirling@ill.eu

Keywords: Neutron Strain Scanning, Phase Specific Strain, 4-Point Bending, Stress Free Lattice Parameter, Duplex Steel

\begin{abstract}
Neutronographic residual stress analysis on multiphase materials is challenging with regard to phase-specific micro residual stresses and to the consideration of an appropriate stress free lattice parameter for meaningful lattice strain calculation. Even in case of randomly textured materials stress analysis becomes more elaborate due to plastic anisotropy effects. According to literature for stress analysis using neutron diffraction lattice planes should be chosen that are less prone to plastic anisotropy. These are the $\{311\}$ austenite and the $\{220\}$ ferrite planes in case of duplex steels. Here, we report about phase-specific in-situ neutron strain scanning at SALSA@ILL, Grenoble during defined 4-point-bending of duplex steel X2CrNiMoN22-5-3 using exactly these two recommended diffraction lines. It is shown that due to the local texture of the bending bars, which was cut from a hot rolled cylindrical rod, strong plastic anisotropy was determined. This effect must be taken into account for diffraction based residual stress analysis to prevent from erroneous stress determination.
\end{abstract}

\section{Introduction}

For non-destructive residual stress analysis for the inside of technical components beside high energy synchrotron X-ray diffraction, neutron diffraction is often the method of choice. In this regard a meaningful measure of the stress / strain free or stress / strain independent lattice parameter $\mathrm{D}_{0}$ is required for calculating lattice strains from the determined interplanar lattice spacings. Different procedures are explained how to provide appropriate data for $\mathrm{D}_{0}$ as e.g. determining the interplanar lattice spacings for the 'stress free state' in an area with negligible (residual) stress or to cut cubes or comb structures out of the material, which in turn leads to a partial and adequate stress release [1]. In case of multi-phase materials, phase specific micro residual stresses can impede these well established procedures since it is questionable if the micro residual stresses will be released during cutting the samples. Assuming that the macro residual stresses are sufficiently released significant phase specific residual stresses might remain and will in consequence affect the local stress free lattice parameter.

For diffraction stress analysis in multiphase materials it is recommended to determine phase specific lattice strains in all phases if the phase content exceeds a volume share of about $10 \%$. By this means macro (residual) stresses can be determined using a rule of mixture and the volume share of the contributing phases as weighting factor. Duplex steels represent typical applicants in 
this field. Here, large amount of austenite phase coexists with ferrite, hence for this gross twophase material neutronographic stress/strain analysis strongly requires consideration of both phases and thus also the determination of appropriate phase specific stress free lattice parameters $\mathrm{D}_{0, \alpha}$ and $\mathrm{D}_{0, \gamma}$. For the assessment of manufacturing processes by means of process induced residual stresses often the separation of macro and micro residual stresses and in this regard the load partitioning on the two phases is of special interest. In this context in [2] the phase specific lattice strains for various $\{\mathrm{hkl}\}$ lattice planes for duplex steel subjected to defined macroscopic elastic and elasto-plastic uniaxial loading were studied using neutron diffraction. It has been shown that apart of the elastic anisotropy plastic anisotropy effects occur due to the fact that crystallites do not deform homogeneously since the deformation depends on the slip systems. Regarding the load partitioning for different lattice planes this plastic anisotropy in part strongly deviates from the elastic behavior. For practical applications the authors recommended to consider only lattice planes for (residual) stress analysis which have no or only a weak anisotropic effect. In accordance with [3,4] for fcc materials the $\{111\},\{311\}$ and $\{422\}$ planes and for bcc materials the $\{110\}$ or $\{211\}$ planes are less prone to plastic anisotropy effects. In preliminary studies on load partitioning in duplex steels we noticed that crystallographic texture might have a strong impact on the plastic anisotropy. However, crystallographic texture was not discussed in the above mentioned works. The knowledge about the impact of crystallographic texture on plastic anisotropy is essential for the planning, the evaluation and assessment of neutronographic stress analysis, not least since semi-finished parts or technical components made of duplex steels often exhibit characteristic textures due to the processing route. To study the effect of texture on the load partitioning behavior for this first approach rectangular shapes bars were extracted from cylindrical rods of duplex steel X2CrNiMoN22-5-3 exhibiting phase fractions of about $50 \%$ ferrite $(\alpha)$ and $50 \%$ austenite $(\gamma)$. For samples subjected to defined $4-$ point-bending using purely elastic and elasto-plastic loading neutronographic strain scanning in both phases was carried out with respect to the coordinate of the bending height on instrument SALSA@ILL, Grenoble. 4-point-bending experiments have the charm that within a single experiment different uniaxial loading states can be studied for different location at the bar. Considering purely elastic and elasto-plastic loading allows for the separation between elastic and plastic anisotropy effects. To provide meaningful data for the phase specific stress free lattice parameters $\mathrm{D}_{0,\{\mathrm{hkl}\}}$ the locally exiting lattice parameters of the sample prior to the loading experiment is determined and applied for local lattice strain calculation.

\section{Material and experimental Procedures:}

The used material is a hot-rolled duplex steel X2CrNiMoN22-5-3 (mat.no. 1.4462) with a diameter of $30 \mathrm{~mm}$ exhibiting a ferrite to austenite ratio of approximately 50:50. The microstructure is shown in Fig. 1 for a cut in longitudinal direction. Metallographic analysis revealed an average grain size in rolling direction (later longitudinal direction) of approx. $74 \mu \mathrm{m}, 6 \mu \mathrm{m}$ in transverse direction and $5 \mu \mathrm{m}$ in normal direction, respectively. The sample geometry is $160 \times 15 \times 10 \mathrm{~mm}$. Figure 2 shows a schematic view of the sample with the assigned coordinate system together with indication of the applied 4-point-bending loading.

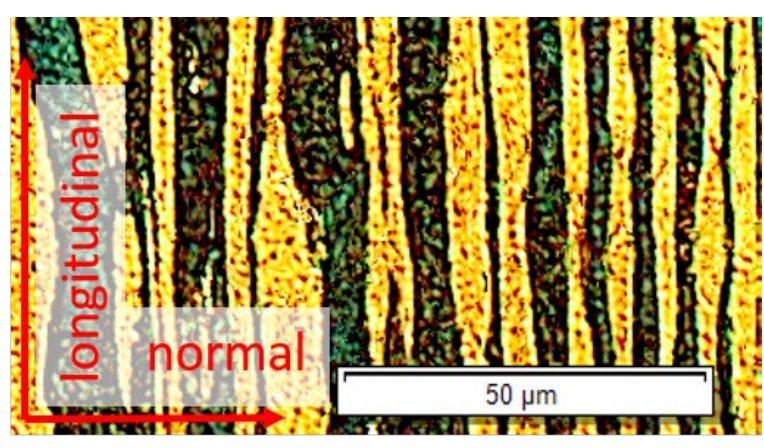

Figure 1: Microstructure of the duplex steel for a section from a cut in longitudinal direction of the used bars, etchant: Behara II, bright regions: austenite, dark regions: ferrite 
In the as received state the duplex steel was de facto stress free as verified by means of hole drilling and X-ray stress analysis. As indicated in the introduction part the bars exhibit a rather strong crystallographic texture in both phases with a gradient over the bending height. Since the bending bars were not exactly cut from the centre of the as delivered steel rod the texture distribution is not symmetric to the mid layer of the bars. Fig. 3 shows as an example the texture distribution for the later compressive loaded layer, the mid layer and for the later tensile loaded layer for both phases for the $\phi 2=45^{\circ}$ sections of the ODF (orientation distribution function) determined by means of X-ray diffraction prior to the neutron diffraction experiment. In total the ODF was determined for 10 locations distributed over the bending height. The ODF cuts

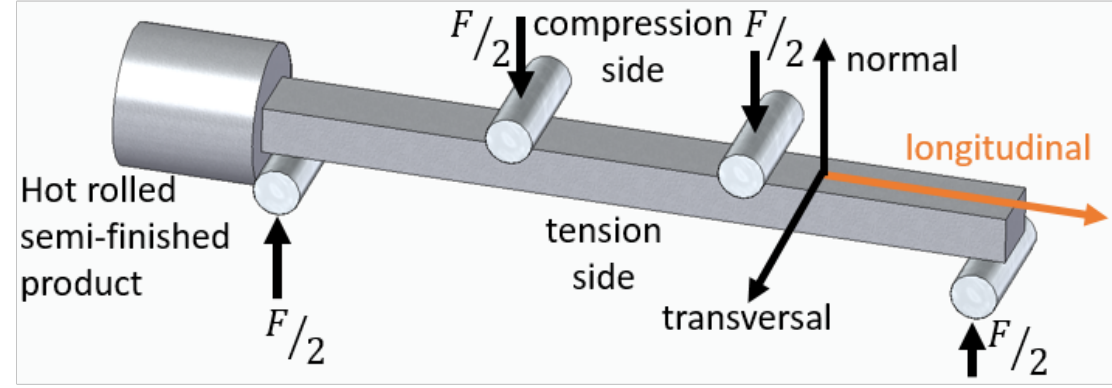

Figure 2: Schematic sketch of the bending bar with indication of the applied coordinate system exemplary shown in Fig. 3 clearly indicate that (a) a gradient in the crystallographic texture exist in both phases and (b) that the sharpness of the texture is much higher in case of the ferrite phase (in particular on the later compressive loaded side).

Neutron strain scanning with respect to the bending height was carried out in all three principal directions, i.e. in longitudinal, transverse and normal direction (see Fig 2) in both phases for various $\{\mathrm{hkl}\}$ planes at the SALSA experiment at ILL [5], Grenoble using a wavelength of $1.6 \AA$. A nominal gauge volume of $0.6 \times 0.6 \times 10 \mathrm{~mm}$ was defined by radial collimators at the primary and secondary beam paths; the $10 \mathrm{~mm}$ axis was always parallel to the longitudinal direction of the bar. In total two ferrite $(\alpha-\mathrm{Fe})$ lattice planes $(\{211\},\{220\})$ and three austenite $(\gamma$-Fe) lattice planes $(\{220\},\{222\},\{311\})$ were measured separately. Due to the rather strong local texture not for all directions interference lines could be recorded with intensities being sufficient for meaningful evaluation of the line positions. That means that the local texture impeded stress determination at all. Here, we only focus on the $\{220\} \alpha$-ferrite and on the $\{311\}$ austenite lattice planes, which are (according to literature) expected to be less prone to plastic anisotropy. Furthermore, we only focus on the strain scanning of the longitudinal component, which corresponds to the loading direction, to study the load partitioning in both phases. The peak fitting was realized using a Gaussian function subsequent to background subtraction. Only measurement locations, where the entire nominal gauge volume is immersed into material are considered. The phase specific values of the stress free lattice parameters $\mathrm{D}_{0,\{\mathrm{hkl}\}}$ were determined on the bending bar prior to the bending loading at the same positions, which were used for later in-situ lattice strain scanning during loading. Since, the $\mathrm{D}_{0}$ scanning was carried out for less measuring points as in case of the in-situ experiments linear interpolation was applied. For in-situ neutron strain scanning during defined bending loading two different load stages were considered: (i) purely elastically deformed $(0.22 \%$ total strain) and (ii) elasto-plastically deformed (approx. $1.5 \%$ total strain). The loading was controlled via the total strain measured by strain gauges at both outer fibres of the bar. The loading stress were assigned using a reference bending stress-strain curve determined on an instrumented universal testing machine prior to the neutron beamtime using the same support distances as given by the 4-point bending device applied at SALSA (see Fig. 2). 


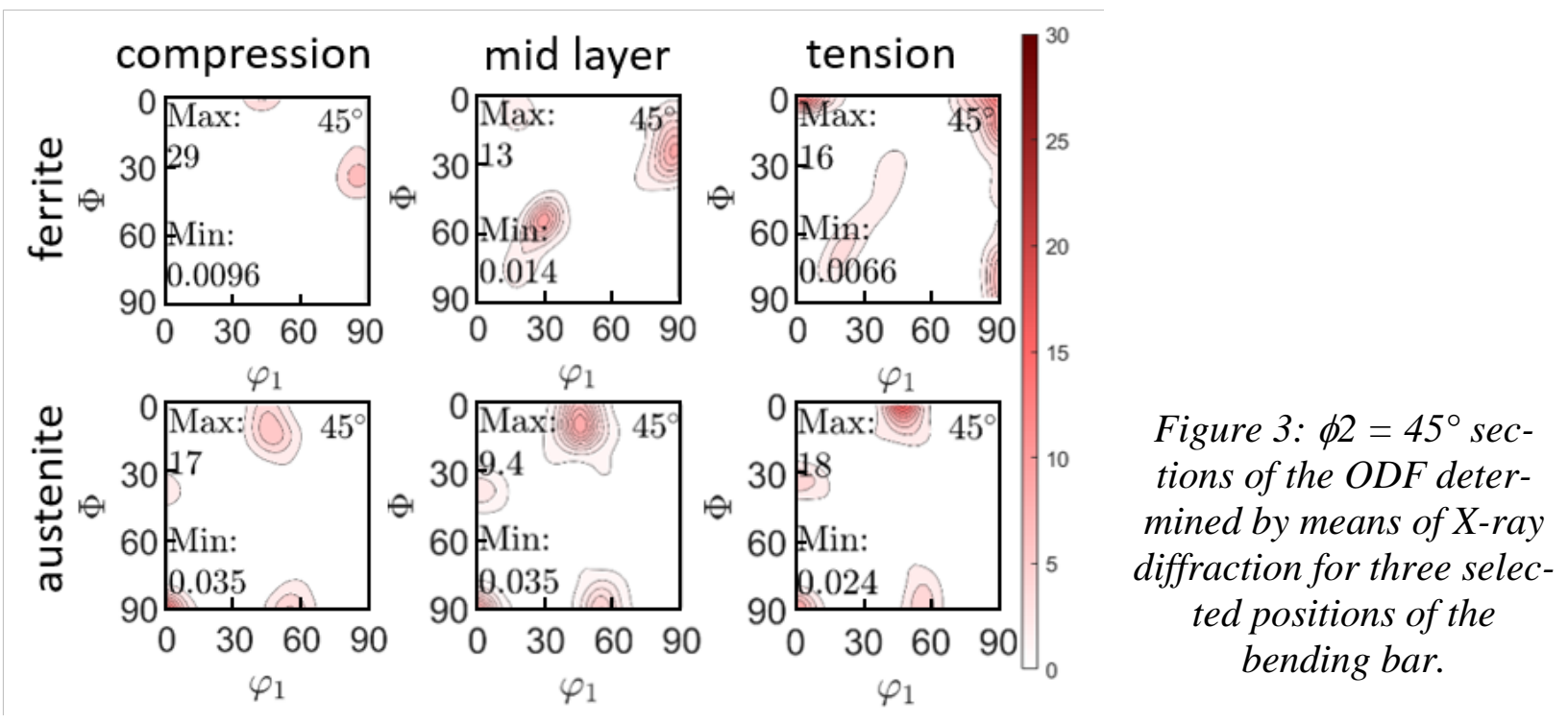

\section{Experimental results and discussion}

Figures 4 (A) and (B) show the interplanar lattice spacings for the initial state of the bending bar determined for the $\{311\} \gamma$-Fe and the $\{220\} \alpha$-Fe lattice planes, respectively. The data indicate that the stress free lattice parameters $\mathrm{D}_{0,\{\mathrm{hkl}\}}$ shows a characteristic distribution presumable due to the processing route (hot rolling) of the rods, which leads to local texture and the generation of phase specific micro residual stresses. On the side with negative coordinates (later compressive loaded side) large errors bars result for the ferrite phase, which is due to local texture distribution and the connected weak intensities of the $\{220\}$ interference lines. Regarding lattice strains that can be assigned to the changes in lattice parameters $D_{0}$, these changes in lattice parameters correspond to rather high strain (up to about 0.0016). Hence, neglecting this initial state might result in erroneous stress data. Consequently, the lattice strains presented in Fig. 4 (C) for the purely elastic case $\left(\varepsilon_{\mathrm{t}}=0.22 \%\right)$ and Fig. $5(\mathrm{~A})$ for the elasto-plastic case $\left(\varepsilon_{\mathrm{t}}=1.5 \%\right)$ are lattice strain differences $\Delta \varepsilon$ calculated with reference to this initial state.
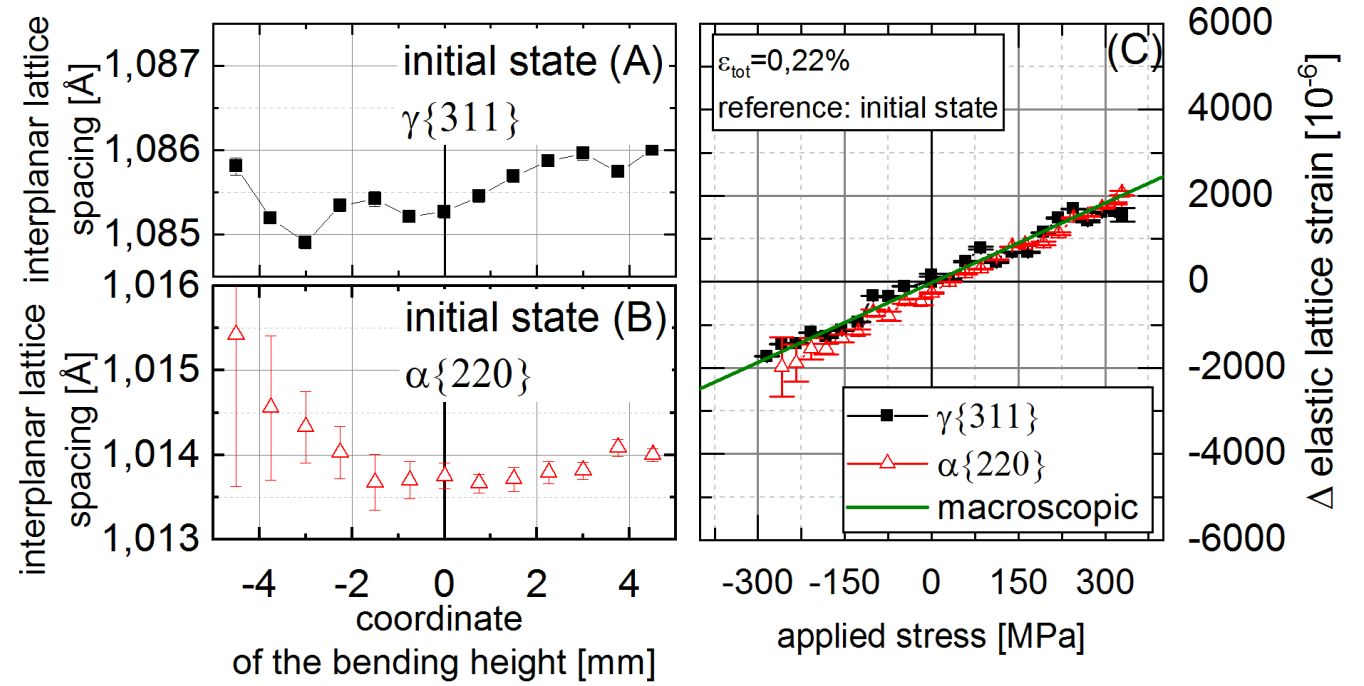

Figure 4: Phase-specific interplanar lattice spacings determined for the in the initial state of the bending bar for (A) the $\{311\}$ austenite lines and (B) for the $\{220\}$ ferrite lines. In (C) the change in lattice strains $\Delta \varepsilon$ with respect to the initial state for the macroscopically purely elastic loading state is shown. For better orientation the macroscopic $\varepsilon$ vs. $\sigma$ distribution is plotted. 
In Fig. 4 (C) the lattice strain difference $\Delta \varepsilon$ for the purely elastic loading vs. the applied stress is shown. For the $\{311\} \gamma$-Fe and the $\{220\} \alpha$-Fe lattice planes almost linear courses are determined. Furthermore, both distributions roughly follow the macroscopic distribution, which can be expected for the orientation factors of $3 \Gamma=0.471$ for the $\{311\}$ austenite and $3 \Gamma=0.75$ for the $\{220\}$ ferrite planes. Both values are close to $3 \Gamma=0.6$, which generally reflects the macroscopic behavior.

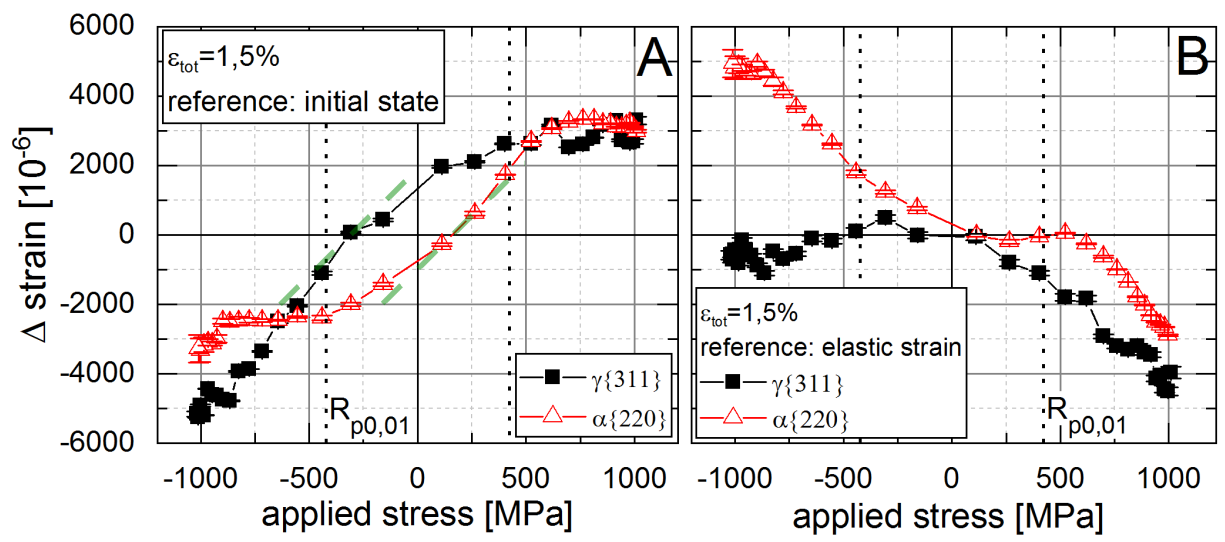

Figure 5: (A) change in phase-specific lattice strains $\Delta \varepsilon$ with respect to the initial state for elasto-plastic loading to $\varepsilon_{t}=1.5 \%$. For better orientation the macroscopic $0.01 \%$ proof stress $R_{p 0.01}$ is added to the plot; (B) change in phase-specific lattice strains $\Delta \varepsilon$ after subtraction of the fictitious elastic materials response for the same loading state.

In Fig. 5 (B) the phase-specific load partitioning for the duplex steel in case of elasto-plastic loading is displayed for the loading with total strains in the outer layers of the bending bar of approx. $1.5 \%$. Again the change in lattice strain with reference to the initial state is presented. In Fig. 5 (B) the same data is plotted as used in Fig. 5 (A), but here the fictitious elastic strain is subtracted for both phases using the trend determined for the purely elastic loading as shown in Fig. 4 (C). Here, the zero passage determined in Fig. 5 (A) is maintained for both phases. By this means the change in phase specific lattice strain plotted in Fig. 5 (B) indicate, when the individual phases start to plastify and by this means, differences in the phase specific materials response can be noticed more conveniently. To provide better orientation the $0.01 \%$ proof stress $\mathrm{R}_{\mathrm{p} 0.01}(423 \mathrm{MPa}$ ) as determined by the macroscopic bending work hardening curve is added. The changes in phase specific lattice strains with respect to the initial state (Fig. 5(A)) indicate that in both phases large deviations to the behavior expected based on the work of [2] occur. In particular for the $\{220\} \alpha$-Fe interference line it is expected that no significant plastic anisotropy exists. However, for both phases strong plastic anisotropy effects occur, which can be explained by the phase-specific texture exhibiting a strong gradient through the bending bar (see Fig. 3). According to [2] the strain response for the $\{220\}$ ferrite lattice planes should follow the trend from the elastic regime. However, in the present case on both sides, i.e. the tensile and the compressive loaded side strong deviations occur, indicating that local phase-specific plastic deformation is strongly affected by the local crystallographic texture. That means that the texture is such that crystallites deform inhomogenously over the cross section of the bars. The reason is that the crystallites are orientated such that slip planes and slip directions are oriented in a way that dislocation gliding during bending loading is promoted in contrast to a more random orientation. In detail this will be studied by means of simulation based on crystal plasticity modelling taking into account the local gradient in crystallographic texture and is part of current research efforts. Here, the results of neutronographic strain scanning will be used for validation of the modelling approach. 
A closer look to the data plotted in Fig. 5 (A) further reveals that the observed behavior is asymmetric when comparing the compressive with the tensile loaded side. The asymmetric phase-specific plastic deformation obviously causes a shift of the neutral fiber in both phases, i.e. in ferrite slightly towards the tensile and in austenite slightly towards the compressive loaded side. Obviously, the neutral fiber resulting for the macroscopic behavior is slightly shifted towards the compressive loaded side under consideration of the volume content, which is about 50:50. This shift of the neutral fiber (characterizing the macroscopic behavior) is nothing odd and can also be explained by means of the texture gradient.

The change in phase specific lattice strains when subtracting the fictitious elastic response supports the above mentioned statements. From this plot it can be clearly derived that for the $\{220\}$ ferrite line a very pronounced plastic anisotropy in tension and in compression can be noticed, while on the tensile loaded side the slope appears to be slightly steeper. In contrast, strong plastic anisotropy can be observed for the $\{311\}$ austenite interference line. On the compressive loaded side the local texture causes that almost no plastic anisotropy occurs. The local crystallographic texture induces a phase specific mechanical response that strongly deviates from literature. Hence, following the recommendations for consideration of the $\{311\}$ austenite and the $\{220\}$ ferrite interference lines will definitely result in erroneous phase specific residual stresses, when the effect of local crystallographic texture on the plastic deformation behavior is unattended during data evaluation and assessment. Hence, in case that texture is expected from the processing route, special care must be taken in regard to phase-specific plastic anisotropy.

\section{Summary}

In-situ neutronographic phase specific strain scanning during elastic and elasto-plastic 4-point bending was performed for duplex steel X2CrNiMoN22-5-3. The strain in load direction was determined for the $\gamma\{311\}$ and the $\alpha\{220\}$ interference lines under consideration of the local initial state of the bending bars prior to plastic deformation as $\mathrm{D}_{0}$ reference. Inconsistent with literature strong plastic anisotropy occurs for both interference lines, while a neglectable effect was expected. However, in literature local texture was not particularly taken into account. In the present case, local crystallographic texture cause this strong plastic anisotropy for interference lines, which are often recommended and applied for local neutron stress analysis. Based on the findings special care on the texture induced plastic anisotropy must be taken for data evaluation and assessment for duplex steels, which often show pronounced texture induced by processing.

\section{Acknowledgements}

The work was partly supported through a joint project funded by the Czech Science Foundation (project No. 16-08803J) and the German Research Foundation (DFG project GI 376/11-1 and HO 3322/4-1) and by the Czech Ministry of Education, Youth and Sports (project no. LM2015050). The authors are grateful for granting beamtime at ILL (proposal No. 1-02-199).

\section{References}

[1] Withers, P. J., Preuss M., Steuwer A., Pang J. W. L., Appl. Crystallogr. 40 (2007), pp. 891904. https://doi.org/10.1107/S0021889807030269

[2] Allen, A. J.; Bourke, M.; David, W. I. F.; Dawes, S.; Hutchings, M. T.; Krawitz, A. D.; Windsor, C. G., ICRS 1989, pp. 78-83

[3] Clausen B., Lorentzen T., Bourke M., Daymond M., Mat. Sci. Eng. A, 259 (1999), pp. 17-

24. https://doi.org/10.1016/S0921-5093(98)00878-8

[4] Pang, J.W.L; Holden, T.M; Mason, T.E., Acta Mat., (1998), pp. 1503-1518. https://doi.org/10.1016/S1359-6454(97)00369-8

[5] Pirling, T. Bruno, G., Withers, P., Mat. Sci. Eng. A, 437 (2006), pp. 139-144. https://doi.org/10.1016/j.msea.2006.04.083 